\title{
Nonlinear Mach-Zehnder interferometer with ultrabroadband squeezed light
}

\author{
D. B. Horoshko, M. I. Kolobov, F. Gumpert, I. Shand, F. König \& M. V. \\ Chekhova
}

To cite this article: D. B. Horoshko, M. I. Kolobov, F. Gumpert, I. Shand, F. König \& M. V. Chekhova (2019): Nonlinear Mach-Zehnder interferometer with ultrabroadband squeezed light, Journal of Modern Optics, DOI: 10.1080/09500340.2019.1674394

To link to this article: https://doi.org/10.1080/09500340.2019.1674394

\section{曲 Published online: 08 Oct 2019.}

\section{Submit your article to this journal $\square$}

Lلll Article views: 100

Q View related articles $\square$

View Crossmark data ¿ 


\title{
Nonlinear Mach-Zehnder interferometer with ultrabroadband squeezed light
}

\author{
D. B. Horoshko (i) a,b , M. I. Kolobov ${ }^{a}$, F. Gumpert ${ }^{c}$, I. Shand ${ }^{d}$, F. König ${ }^{d}$ and M. V. Chekhova ${ }^{c, e}$ \\ a Univ. Lille, CNRS, UMR 8523 - PhLAM - Physique des Lasers Atomes et Molécules, Lille, France; ${ }^{\text {b } B . ~ I . ~ S t e p a n o v ~ I n s t i t u t e ~ o f ~ P h y s i c s, ~ N A S B, ~ M i n s k, ~}$

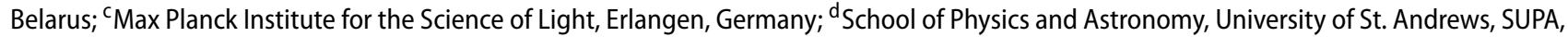 \\ St. Andrews, UK; ${ }^{e}$ Department of Physics, M. V. Lomonosov Moscow State University, Moscow, Russia
}

\begin{abstract}
We study both theoretically and experimentally the interference pattern in a nonlinear Mach-Zehnder interferometer formed by two aperiodically-poled crystals, where broadband squeezed light is generated by both crystals via parametric down-conversion with a common quasimonochromatic pump. This configuration is important for measuring the squeezing produced by the first crystal and also for measuring a small phase shift introduced by a sample between the crystals. On the basis of the approximate quantum Rosenbluth formula for each crystal we develop an analytic model for the field evolution in the interferometer. We report an experimental observation of the interference fringes, caused by the dispersion of the generated PDC waves in both crystals forming the interferometer. We observe a displacement of the interference pattern caused by a sample between the crystals and infer the phase shift within a band of $20 \mathrm{~nm}$. The experimental data are in a good agreement with the predictions of the developed model, up to imperfections of the samples.
\end{abstract}

\section{ARTICLE HISTORY}

Received 27 May 2019

Accepted 25 September 2019

KEYWORDS

Squeezed states; parametric down-conversion; nonlinear interferometer

\section{Introduction}

High-gain parametric down-conversion (PDC) in aperiodically poled crystals leads to the generation of bright squeezed vacuum twin beams with massively broadened spectra. Such twin beams are of considerable interest for quantum photonics and quantum information, due to their multimode structure, ultrashort correlation times and, most importantly, non-classical features such as broadband squeezing (1-3). Bright squeezed vacuum is a macroscopic quantum object exhibiting photon-number entanglement (4) and suitable for quantum metrology. In particular, squeezed vacuum is known to increase the phase sensitivity of an interferometer: by feeding it into the empty port of a Mach-Zehnder interferometer one can overcome the shot-noise limit and under ideal conditions reach the ultimate Heisenberg limit (5). Moreover, by producing squeezed vacuum in an optical parametric amplifier (for instance, a nonlinear crystal) and then sending it to another optical parametric amplifier one obtains a nonlinear Mach-Zehnder interferometer $(6,7)$. This scheme, also known as an SU(1,1) interferometer, not only enables sub-shot-noise phase measurement (ideally reaching the Heisenberg limit), but also provides tolerance to inefficient detection. Broadband squeezing in combination with a nonlinear interferometer would allow one to perform sub-shot-noise phase measurement in a wide band of frequencies, comprising tens of $\mathrm{THz}$.

In a recent work (8), broadband bright twin beams have been generated in an aperiodically poled lithium niobate crystal and their sub-picosecond correlation times have been observed. The observation of quadrature squeezing is, however, more challenging. Standard continuous-variable homodyne detection is inapplicable in this case because of a very large squeezing bandwidth, above $40 \mathrm{THz}$ in the experiment (8), which surpasses the photodetector bandwidth by several orders of magnitude. Temporal imaging technique $(9,10)$ is widely used for matching the bandwidth of light to that of the photodetector, but the application of this technique to squeezed light requires a parametric time lens with high conversion efficiency $(11,12)$, which is technologically challenging. Pulsed homodyne detection (13) requires frequency tailoring of the local oscillator, which is quite demanding, given the complicated spectrum of the twin beams.

Recently a method was proposed (14) to measure quadrature squeezing by using a Mach-Zehnder

CONTACT D. B. Horoshko dmitri.horoshko@univ-lille.fr $=$ Univ. Lille, CNRS, UMR 8523 - PhLAM - Physique des Lasers Atomes et Molécules, F-59000 Lille, France; B. I. Stepanov Institute of Physics, NASB, Nezavisimosti Ave. 68, 220072 Minsk, Belarus

This article has been republished with minor changes. These changes do not impact the academic content of the article.

The data that support the findings of this study are openly available in Zenodo at https://doi.org/10.5281/zenodo.3520515 
nonlinear interferometer $(6,7)$. The mean number of photons at the output of the second amplifier scales then as the variance of a certain quadrature at the output of the first amplifier; the phase of the quadrature under test depends on the phases of the pump, signal, and idler photons inside the interferometer. This 'optical homodyne' technique will be extremely helpful for the investigation of broadband squeezing for twin beams generated in aperiodically poled crystals.

The aim of this article is to consider a nonlinear interferometer consisting of two aperiodically poled crystals placed into a common pump and generating broadband twin beams through high-gain PDC. The long-term motivation for considering this configuration is twofold: (i) measuring the broadband squeezing, produced by the first crystal (14), to determine the frequency band available for quantum operations, and (ii) measuring the phase shift introduced by a sample between the crystals in a wide band of frequencies $(15,16)$. Our short-term objectives are building an analytic model of such an interferometer and observing the interference fringes as well as their displacement caused by the sample.

The paper has the following structure. In Section 2 we derive the equations describing the nonlinear interferometer consisting of two aperiodically poled crystals. Section 3 presents the analytic modelling of the spectra, both for a single crystal and for a combination of two crystals. Section 4 describes the experiment where the nonlinear interference fringes were observed and a phase shift measured. The last Section 5 concludes the paper.

\section{Nonlinear interferometer}

We consider a nonlinear Mach-Zehnder interferometer (MZI) composed of two aperiodically poled crystals $(1,2,8)$, as shown in Figure 1. The signal and idler produced in the first crystal arrive at the second crystal with a frequency-dependent phase shift $\phi(\Omega)$, which can correspond to passing through a dispersive sample or just to a time delay in the free space.
In each crystal, collinear type-I PDC annihilates one pump photon with the frequency $\omega_{\mathrm{p}}$ and creates two photons with the same direction and polarization, and frequencies $\omega_{0}+\Omega$ and $\omega_{0}-\Omega$, where $\omega_{0}=\omega_{\mathrm{p}} / 2$. The phase mismatch for this process has the form $\Delta(\Omega)=$ $k_{\mathrm{p}}-k(\Omega)-k(-\Omega)$, where $k_{\mathrm{p}}$ is the wave vector of the pump wave, assumed to be an undepleted quasimonochromatic plane wave, and $k(\Omega)$ is the wave vector of the down-converted wave at the frequency $\omega_{0}+\Omega$. In general there is no phase matching at degeneracy, $k_{p} \neq$ $2 k_{0}$, where $k_{0}=k(0)$. Let us direct the $z$ axis along the propagation of the waves, placing the origin on the front edge of the first crystal and denoting its length by $L_{1}$. For the description of the field we use the sideband photon annihilation operator $a(\Omega, z)$ corresponding to frequency $\omega_{0}+\Omega$ and position $z$, so that the field operator (in the photon-flux units) is

$$
E^{(+)}(t, z)=\int a(\Omega, z) \mathrm{e}^{i\left(k_{0} z-\left(\omega_{0}+\Omega\right) t\right)} \mathrm{d} \Omega
$$

The field transformations in both crystals have the forms of the Bogoliubov transformations

$$
\begin{aligned}
a\left(\Omega, L_{1}\right)= & U_{1}(\Omega) a(\Omega, 0)+V_{1}(\Omega) a^{\dagger}(-\Omega, 0), \\
a\left(\Omega, z_{\text {out }}\right)= & U_{2}(\Omega) a\left(\Omega, L_{1}+d\right) \\
& +V_{2}(\Omega) a^{\dagger}\left(-\Omega, L_{1}+d\right),
\end{aligned}
$$

where $d$ is the distance between the crystals, $z_{\text {out }}=L_{1}+$ $d+L_{2}$ is the position of the second crystal output. The functions $U_{n}(\Omega)$ and $V_{n}(\Omega)$, where $n$ denotes the crystal number, satisfy the relations $\left|U_{n}(\Omega)\right|^{2}-\left|V_{n}(\Omega)\right|^{2}=1$ and $U_{n}(\Omega) / V_{n}(\Omega)=U_{n}(-\Omega) / V_{n}(-\Omega)$, required by the unitarity of the field transformation. We denote the phase acquired between the crystals by the field component at the frequency $\omega_{0}+\Omega$ as $\phi(\Omega)$. Writing $a\left(\Omega, L_{1}+d\right)=$ $a\left(\Omega, L_{1}\right) e^{i \phi(\Omega)}$, we obtain from Equations (2) and (3) the total input-output field transformation as

$$
a\left(\Omega, z_{\text {out }}\right)=U_{\text {int }}(\Omega) a(\Omega, 0)+V_{\text {int }}(\Omega) a^{\dagger}(-\Omega, 0),
$$

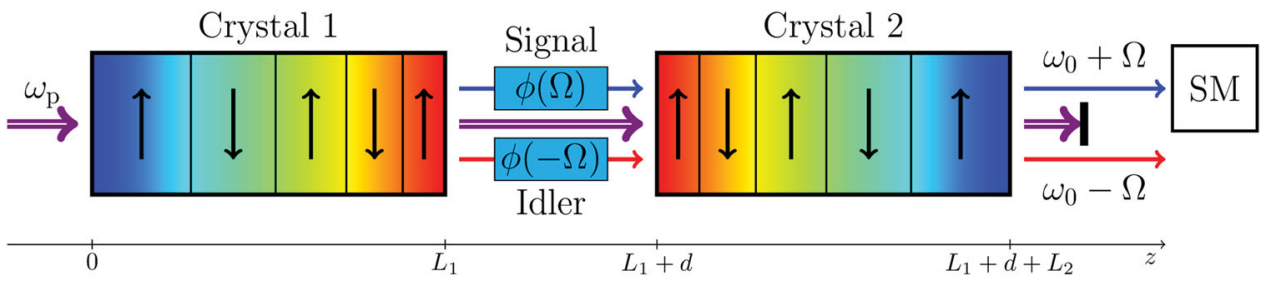

Figure 1. Nonlinear interferometer formed by two aperiodically poled crystals. Both crystals are pumped by a strong coherent wave at frequency $\omega_{p}$ and are quasi-phase-matched for parametric downconversion in a broad range of frequencies. The signal and the idler waves from the first crystal acquire a frequency-dependent phase shift $\phi(\Omega)$ and then pass through the second crystal, where they are further amplified or attenuated depending on the relative phase with the pump. The power spectrum of the signal is measured at the output of the second crystal by a spectrometer (SM). The colour of the crystal layers shows schematically the signal frequency phase-matched at the given position. 
where

$$
\begin{aligned}
U_{\text {int }}(\Omega)= & U_{2}(\Omega) U_{1}(\Omega) \mathrm{e}^{i \phi(\Omega)} \\
& +V_{2}(\Omega) V_{1}^{*}(-\Omega) \mathrm{e}^{-i \phi(-\Omega)} \\
V_{\text {int }}(\Omega)= & U_{2}(\Omega) V_{1}(\Omega) \mathrm{e}^{i \phi(\Omega)} \\
& +V_{2}(\Omega) U_{1}^{*}(-\Omega) \mathrm{e}^{-i \phi(-\Omega)}
\end{aligned}
$$

are the Bogoliubov coefficients for the entire interferometer.

Each transformation can be parametrized by four real functions of the frequency detuning (17):

$$
\begin{aligned}
r_{n}(\Omega) & =\ln \left(\left|U_{n}(\Omega)\right|+\left|V_{n}(\Omega)\right|\right), \\
\psi_{n}^{0}(\Omega) & =\frac{1}{2} \arg \left[U_{n}^{-1}(\Omega) V_{n}(\Omega)\right], \\
\psi_{n}^{L}(\Omega) & =\frac{1}{2} \arg \left[U_{n}(\Omega) V_{n}(-\Omega)\right], \\
\kappa_{n}(\Omega) & =\frac{1}{2} \arg \left[U_{n}(\Omega) U_{n}^{-1}(-\Omega)\right],
\end{aligned}
$$

where $r_{n}(\Omega)$ is known as the squeezing parameter at frequency $\omega_{0}+\Omega$, while $\psi_{n}^{0}(\Omega)$ and $\psi_{n}^{L}(\Omega)$ are the input and the output squeezing angles, respectively.

The power spectrum of the signal wave at the interferometer output $S\left(\omega_{0}+\Omega\right)=\left|V_{\text {int }}(\Omega)\right|^{2}$ is readily obtained from Equations (6)-(9) as

$$
\begin{aligned}
& S\left(\omega_{0}+\Omega\right) \\
& \quad=\sinh ^{2}\left(r_{2}+r_{1}\right) \cos ^{2}\left(\psi_{1}^{L}-\psi_{2}^{0}+\phi_{S}\right) \\
& \quad+\sinh ^{2}\left(r_{2}-r_{1}\right) \sin ^{2}\left(\psi_{1}^{L}-\psi_{2}^{0}+\phi_{S}\right),
\end{aligned}
$$

where $\phi_{S}(\Omega)=(\phi(\Omega)+\phi(-\Omega)) / 2$ is the frequencysymmetrized phase shift. Note that in general $r_{1}(\Omega) \neq$ $r_{2}(\Omega)$, because the gain values in the crystals may be different. The first obvious consequence of Equation (11) is the invariance of the spectrum to the distance between the crystals when the phase shift is due to propagation in the free space. Indeed, in this case $\phi(\Omega)=\Omega d / c$ and, as a consequence, $\phi_{S}(\Omega)=0$ for any distance $d$ between the crystals.

An interesting case corresponds to a parametric gain of the second crystal being significantly higher than that of the first one, $\exp \left(2 r_{2}\right) \gg \exp \left(2 r_{1}\right) \gg 1$. In this case Equation (11) can be rewritten as $S\left(\omega_{0}+\Omega\right) \approx$ $\mathrm{e}^{2 r_{2}} S_{1}\left(\omega_{0}+\Omega\right) / 4$, where

$$
\begin{aligned}
& S_{1}\left(\omega_{0}+\Omega\right) \\
& \quad=\mathrm{e}^{2 r_{1}(\Omega)} \cos ^{2}\left[\psi_{1}^{L}(\Omega)-\psi_{2}^{0}(\Omega)+\phi_{S}(\Omega)\right] \\
& \quad+\mathrm{e}^{-2 r_{1}(\Omega)} \sin ^{2}\left[\psi_{1}^{L}(\Omega)-\psi_{2}^{0}(\Omega)+\phi_{S}(\Omega)\right],
\end{aligned}
$$

which is similar to the homodyne photocurrent spectrum with $\psi_{2}^{0}-\phi_{S}$ playing the role of the local oscillator phase. On the one hand, when the second crystal is calibrated, i.e. the values of $r_{2}(\Omega)$ and $\psi_{2}^{0}(\Omega)$ are known with high precision, observation of an interferometric picture in accordance with Equation (11) can be interpreted as 'ultrabroadband nonlinear homodyne measurement' (14) of the squeezed light produced by the first crystal. On the other hand, Equation (11) can be used for the measurement of small phase shifts introduced by a dispersive sample between the crystals. It is clear that the spectrum contains fringes, determined by the angle $\psi_{1}^{L}(\Omega)-\psi_{2}^{0}(\Omega)$, and a small addition $\phi_{S}(\Omega)$ results in a shift of these fringes, which allows one to measure it. It is important, however, that the fringes are resolved by the spectrometer monitoring the signal output. To this end the variation of the angle $\psi_{1}^{L}(\Omega)-\psi_{2}^{0}(\Omega)$ should be made as slow as possible by a proper crystal design.

In the next section we do the analytic modelling of PDC in an aperiodically poled crystal and show how frequency dependence of the angle $\psi_{1}^{L}(\Omega)-\psi_{2}^{0}(\Omega)$ can be made rather weak, while in Section 5 we describe an experimental observation of nonlinear interference in such specially designed crystals.

\section{Analytic modelling of aperiodically poled crystals by means of the quantum Rosenbluth formula}

\subsection{General formalism}

In this section we consider one of the crystals forming the nonlinear interferometer, described in the previous section. We omit the subscript $n$ denoting the crystal number in the major part of formulas to simplify them, but restore it when necessary. The field transformation in an aperiodically poled crystal with the poling profile $K(z)$ is described approximately by the Bogoliubov coefficients, given by the 'quantum Rosenbluth formula' $(1,2)$ :

$$
\begin{aligned}
& U(\Omega)=\mathrm{e}^{\pi \nu(\Omega)} \mathrm{e}^{i\left(k(\Omega)-k_{0}\right) L}, \\
& V(\Omega)=\sqrt{\mathrm{e}^{2 \pi \nu(\Omega)}-1} \mathrm{e}^{-2 i \xi(\Omega)+i\left(k(\Omega)-k_{0}\right) L+i \varphi_{A},}
\end{aligned}
$$

with the frequency-dependent Rosenbluth parameter $v(\Omega)$ being defined as

$$
\nu(\Omega)=\frac{|\gamma|^{2}}{\left|K^{\prime}\left(z_{p m}(\Omega)\right)\right|} .
$$

Here $\gamma$ is the coupling constant, including the pump amplitude, and $z_{p m}(\Omega)$ is the point of perfect phase matching for the frequency $\Omega$, defined by the equation $K\left(z_{p m}\right)=\Delta(\Omega)$. 
The two phases in Equation (14) are defined as

$$
\xi(\Omega)=-\frac{1}{2} \int_{z_{\text {in }}}^{z_{p m}(\Omega)}(\Delta(\Omega)-K(z)) \mathrm{d} z,
$$

where $z_{i n}$ is the input coordinate of the crystal, i.e. $z_{i n, 1}=$ 0 for the first crystal and $z_{i n, 2}=L_{1}+d$ for the second one, while $\varphi_{A}=\arg (i \gamma)+\varphi_{1}$, where $\varphi_{1}$ is the gaindependent phase, which can be approximated for $v \leq 2$ as $\varphi_{1} \approx-v+v^{2} / 4$.

In many practical cases the phase mismatch function can be approximated by the second-order polynomial in frequency:

$$
\Delta(\Omega) \approx-\alpha\left(\frac{\Omega}{\omega_{0}}\right)^{2}+\beta .
$$

If this approximation holds, then the angle $\xi(\Omega)$, and as consequence, the input and output squeezing angles $\psi^{0}(\Omega)$ and $\psi^{L}(\Omega)$ can be made quadratic in frequency by employing the quadratic-hyperbolic shape of the aperiodic poling profile (2):

$$
\begin{aligned}
K(z)= & -\left(\frac{\Omega^{0} \Omega^{L}}{\omega_{0}}\right)^{2} \\
& \times \frac{\alpha}{\left[\Omega^{L}-\left(\Omega^{L}-\Omega^{0}\right)\left(z-z_{i n}\right) / L\right]^{2}}+\beta,
\end{aligned}
$$

where $\Omega^{0}$ and $\Omega^{L}$ are the frequency detunings perfectly phase matched at the crystal input and output, respectively. The point of perfect phase matching for such a profile depends on the frequency as

$$
z_{p m}(\Omega)=z_{i n}+\frac{\Omega^{0} \Omega^{L}}{\Omega^{L}-\Omega^{0}}\left(\frac{1}{\Omega^{0}}-\frac{1}{\Omega}\right) L,
$$

Performing the integration in Equation (16), we obtain the angle $\xi(\Omega)$, which gives us by Equations (13), (14), (8), (9) the two squeezing angles as follows:

$$
\begin{aligned}
& \psi^{0}(\Omega)=-\frac{\alpha L}{2 \omega_{0}^{2}} \frac{\Omega^{L}\left(\Omega-\Omega^{0}\right)^{2}}{\Omega^{L}-\Omega^{0}}+\frac{\varphi_{A}}{2}, \\
& \psi^{L}(\Omega)=-\frac{\alpha L}{2 \omega_{0}^{2}} \frac{\Omega^{0}\left(\Omega-\Omega^{L}\right)^{2}}{\Omega^{L}-\Omega^{0}}+\frac{\varphi_{A}^{\prime}}{2},
\end{aligned}
$$

where $\varphi_{A}^{\prime}=\varphi_{A}+\alpha L \Omega^{0} \Omega^{L} / \omega_{0}^{2}-\beta L$. We see, that these angles are quadratic functions of the frequency, as required. As consequence, the angle $\psi_{1}^{L}(\Omega)-\psi_{2}^{0}(\Omega)$ entering Equation (12), is also a quadratic function of the frequency, and can, in principle, be compensated by a passive dispersive element introduced into the signal beam between the crystals. In the experiment described in Section 4 this compensation was not performed, and the major part of the fringes in the output spectrum was not resolved by the spectrometer, because the oscillations appeared on a very low frequency scale.

Substituting Equation (18) into Equation (15) we obtain the Rosenbluth parameter for the quadratichyperbolic profile:

$$
v(\Omega)=\frac{\nu^{0}}{2} \frac{\Omega^{L} \Omega^{0}\left(\Omega^{L}-\Omega^{0}\right)}{\Omega^{3}},
$$

where we have introduced the normalized pump intensity,

$$
\nu^{0}=\frac{|\gamma|^{2} L}{|K(0)-K(L)|}=\frac{|\gamma|^{2} L}{\alpha} \frac{\omega_{0}^{2}}{\left|\left(\Omega^{L}\right)^{2}-\left(\Omega^{0}\right)^{2}\right|},
$$

which has a physical meaning of the Rosenbluth parameter for the linear profile, providing a quasi-phasematching in the same frequency band for the given crystal length. $v^{0}$ corresponds roughly to $v(\Omega)$ at the centre of the signal spectrum.

\subsection{Spectrum of a single crystal}

Let us start the study of the interferometer with the spectrum of the first crystal alone. We choose (as in the experiment described in the next section) a crystal of $5 \% \mathrm{MgO}$-doped congruent aperiodically poled $\mathrm{LiNbO}_{3}$ of length $L=5 \mathrm{~mm}$, pumped with relatively long $(\sim 10$ ps) pulses at the wavelength $\lambda_{p}=532 \mathrm{~nm}$. The quadratic approximation of the phase mismatch for this case gives $\alpha=-\Delta^{\prime \prime}(0) \omega_{0}^{2} / 2=735 \mathrm{rad} / \mathrm{mm}$, and $\beta=\Delta(0)=901$ $\mathrm{rad} / \mathrm{mm}$. The signal band is chosen to be from $1.25 \omega_{0}$ to $1.5 \omega_{0}$, and the idler band from $0.5 \omega_{0}$ to $0.75 \omega_{0}$. This corresponds to signal wavelengths of 709-851 nm, and to idler wavelengths of 1419-2128 nm. The corresponding detunings are $\Omega^{L}=0.25 \omega_{0}$ to $\Omega^{0}=0.5 \omega_{0}$.

The spectrum of the signal field of one crystal, calculated as

$$
S\left(\omega_{0}+\Omega\right)=|V(\Omega)|^{2},
$$

is shown in Figure 2(a) for $v_{1}^{0}=0.01$. It is the same for both orientations of the crystal. We see that the Rosenbluth approximation (red dashed line) gives a good description of the spectrum, except for the 'ripples' having a quasi-period about $2 \mathrm{~nm}$ in the vicinity of $770 \mathrm{~nm}$. The corresponding idler spectrum is shown in Figure 2(b).

For comparison, we show in Figure 2(c,d) the same spectra for higher pump intensity, corresponding to $v^{0}=$ 0.2 . The width of the spectrum reduces as the gain increases, in contrast to the case of a uniform crystal (18).

The physical meaning of the absolute value of the spectrum can be understood as the ratio of the total number of photons in the pump coherence time $\Delta t=6$ ps to 

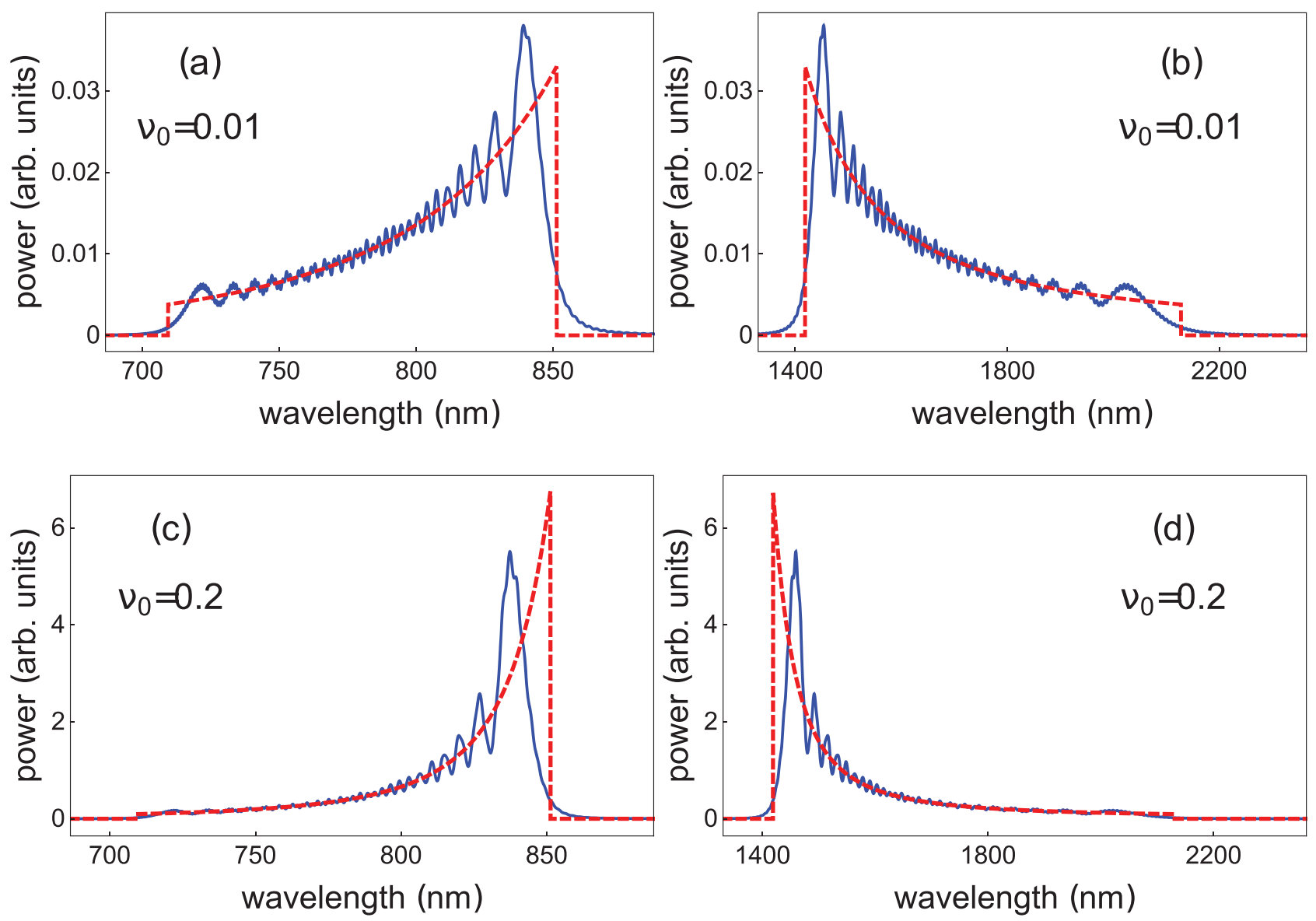

Figure 2. Signal $(a, c)$ and idler (b,d) spectra of aperiodically poled crystal with a quadratic-hyperbolic poling profile: numerical solution of the wave equation (solid line) and the quantum Rosenbluth approximation, Equations (13), (14) (dashed line).

the time-bandwidth product $\Delta t \Delta \Omega / 2 \pi=420$, where $\Delta \Omega=2 \pi \times 70 \mathrm{THz}$ is the signal bandwidth.

\subsection{Nonlinear interferometer}

Now we consider the full interferometer shown in Figure 1, where both crystals have quadratic-hyperbolic profiles, defined by Equation (18), and the first crystal is phase matched from $\Omega_{1}^{0}=0.5 \omega_{0}$ to $\Omega_{1}^{L}=0.25 \omega_{0}$, as above, while the second crystal is its inverted copy, i.e. it is phase matched from $\Omega_{2}^{0}=0.25 \omega_{0}$ to $\Omega_{2}^{L}=0.5 \omega_{0}$. In this configuration the first crystal has a poling profile with the increasing vector $K_{1}(z)$, it starts with long periods and ends with short ones, while the second crystal has a decreasing poling profile $K_{2}(z)$, which starts with short periods and ends with long ones. Putting $\phi_{S}(\Omega)=0$, corresponding to equal delays in the signal and the idler channels, we obtain for the angle in Equation (12)

$$
\psi_{1}^{L}(\Omega)-\psi_{2}^{0}(\Omega)=2 \alpha L \frac{\left(\Omega-0.25 \omega_{0}\right)^{2}}{\omega_{0}^{2}}+\varphi_{I D}
$$

where $\varphi_{I D}$ is a constant phase, specific for the considered configuration.

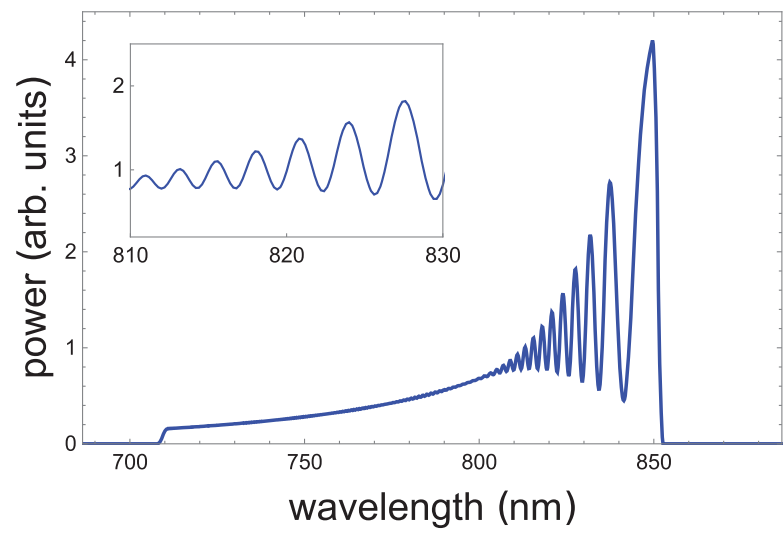

Figure 3. Signal spectrum at the output of the nonlinear MZI given by the quantum Rosenbluth formula for the configuration shown in Figure 1, where the poling profile is increasing in the first crystal and decreasing in the second one.

We choose the normalized pump intensity $v_{1}^{0}=0.01$ for the first crystal and $v_{1}^{0}=0.05$ for the second one. The spectrum $S(\Omega)$ for this configuration is shown in Figure 3, where it is convolved with a Gaussian filter of standard deviation $0.72 \mathrm{~nm}$, corresponding to the 
resolution $1.2 \mathrm{~nm}$ of the spectrometer used for the detection of the visible light in the experiment (see the next section).

The general shape of this spectrum can be understood from simple considerations. The growth of power with the wavelength is characteristic for the quadratichyperbolic profile and is present in the spectra of individual crystals in Figure 2. The oscillations in the spectrum are caused by dispersion experienced by a wave of given frequency between its generation in the first crystal and amplification in the second one. For the longest wavelengths the oscillation frequency is minimal, since this wave is generated at the very end of the first crystal and amplified at the very beginning of the second crystal, so that it does not experience dispersion at all (see red coloured areas in Figure 1). For the shortest wavelengths the effect is opposite: they propagate through the major parts of both crystals and are highly dispersed, which results in rapid spectral oscillations, washed out by the finite resolution bandwidth of the spectrometer.

From the inset of Figure 3 we see that between 810 and $830 \mathrm{~nm}$ there are about 7 periods of the interference pattern, which have been identified in the experiment, described in the next section.

\section{Experiment}

\subsection{Observation of broadband nonlinear interference}

In the experiment, we used the same samples produced by Gooch and Housego as in Ref. (8), the poling period designed to follow the quadratic-hyperbolic dependence (18). Unfortunately, at present the technology of producing aperiodically poled crystals is not yet well developed. Detailed investigation of the samples showed that the actual profile $K(z)$ had deviations from this dependence, up to values of $10 \mathrm{~mm}^{-1}$. Moreover, although the samples had a thickness of $500 \mu \mathrm{m}$, the poling was pronounced only up to a depth of about $100 \mu \mathrm{m}$ from the surface and even within this range, it was not uniform. Because of these imperfections, the spectra varied considerably depending on the sample position, as well as from sample to sample. In particular, the positions of the 'ripples' in the experimental spectra were never reproducible and did not correspond to the ones expected from the theory.

Figure 4 shows the spectra of the signal (left) and idler (right) beams obtained by pumping a single aperiodically poled crystal with 18 ps pulses at $532 \mathrm{~nm}$, the repetition rate being $1 \mathrm{kHz}$ and the mean power $20.8 \mathrm{~mW}$. The spectra were measured with the spectrometers AvaSpecULS3648 (for the signal beam) and NIR QUest256 (for the idler beam). The pump was focused into the crystal with a waist of $170 \mu \mathrm{m}$ full width at half maximum (FWHM). Under this relatively high-gain PDC, the spectrum of signal/idler beams covered a bandwidth of about $25 \mathrm{THz}$ FWHM, which is close to the case shown in the right panel of Figure 2. While the signal beam occupies the accessible range between $725 \mathrm{~nm}$ and $825 \mathrm{~nm}$, the idler one is in a more 'difficult' range 1500-2000 nm. Therefore we observed interference by measuring the spectrum of the signal beam. The observed signal spectrum is rather symmetric and centred at about $775 \mathrm{~nm}$, in contrast to the theoretically predicted spectra in Figure 2. We explain this difference by misalignment in the signal collection system. Note that the idler spectrum is closer to the shape predicted by the theory.

Further, we have placed two aperiodically poled samples of lithium niobate into the common pump beam in such a way that the poling profile was increasing in the first crystal and decreasing in the second one, as shown in Figure 1. The spectra of the first crystal (further called 'the source') and the second one (further called 'the analyser') are shown in Figure 5 by blue dashed and green dotted lines, respectively. Due to the imperfections in the poling, they differ both from each other and from the one presented above (Figure 4). Nevertheless, they overlap over a rather broad spectral band $(810-830 \mathrm{~nm})$, which enables the observation of the nonlinear interference.

Indeed, the spectrum of the nonlinear interferometer (red solid line in Figure 5) shows regular interference fringes, differing considerably from the 'ripples' observed in the spectra of the source and analyser taken separately. Within the range where the spectra of both crystals are overlapping, there are about 7 periods, as predicted by the theory in the previous section.

Extraction of the amount of squeezing from the interference pattern requires a calibration procedure (14) including, in particular, the blocking of only signal or only idler beam inside the nonlinear interferometer. Under our experimental conditions, with both crystals placed into the same pump beam, performing such a procedure is difficult. In addition, the crystals were uncoated, which leads to about $30 \%$ reflection loss of the pump inside the interferometer. The measurement of quadrature squeezing is therefore posed as the next goal of our experiments.

\subsection{Measurement of the phase}

As was mentioned above, a nonlinear interferometer can be used for measuring a phase shift experienced by light passing through a sample. A phase measurement was performed for a plane-parallel BK7 glass slide of thickness $l_{s}=4 \mathrm{~mm}$, inserted between the two crystals of Figure 1, 

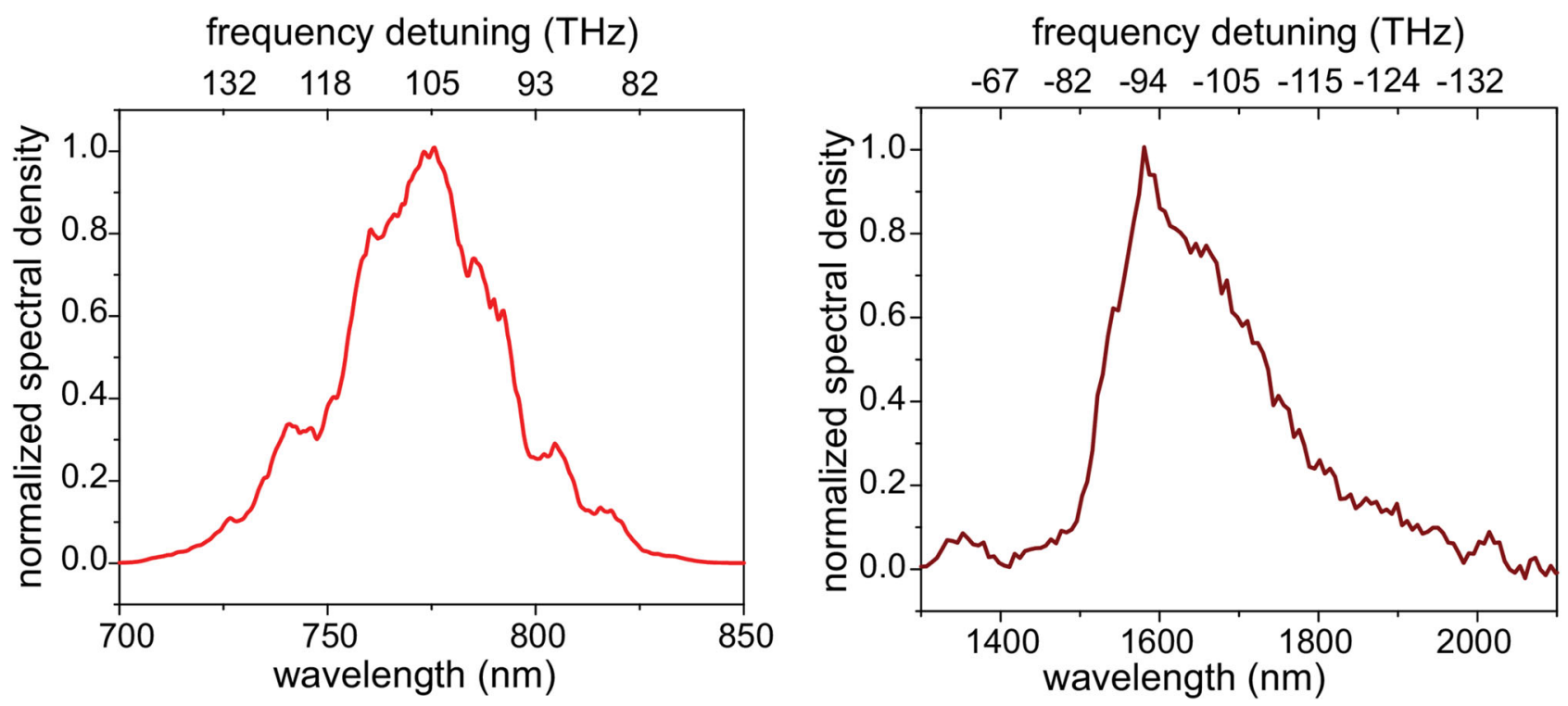

Figure 4. Spectra of the signal (left) and idler (right) beams generated by one aperiodically poled crystal, as observed in the experiment.

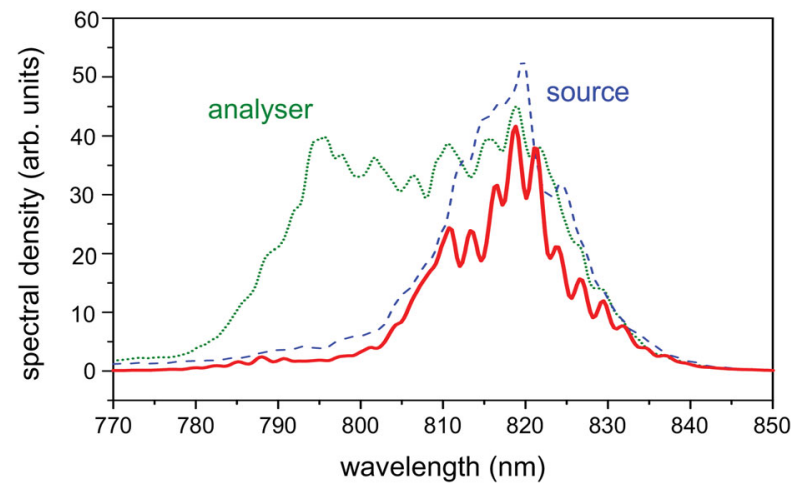

Figure 5. The experimental spectrum (solid line) obtained for the configuration of the nonlinear interferometer shown in Figure 1: the first crystal has the poling profile increasing and the second one, decreasing. The spectra of the first crystal (source) and the second one (analyser) are shown with the dashed and dotted lines, respectively. The oscillations between 810 and $830 \mathrm{~nm}$ are a result of interference and are in a good correspondence with the theoretically predicted fringes shown in Figure 3.

so that pump, signal and idler beams all passed through. This resulted in a phase change of the PDC phase of $\phi_{\mathrm{PDC}}(\Omega)=[k(\Omega)+k(-\Omega)-k(0)] l_{\mathrm{s}}$. The phase $\phi_{\mathrm{PDC}}$ changed the interference fringes in the spectrum after the second crystal. From each measured spectrum, we reconstructed the spectral phase without fitting. Taking the phase difference due to the glass slide, we obtained $\phi_{\text {PDC }}$ up to the usual $2 \pi$ ambiguity. In Figure 6 it is compared with the phase predicted by the Sellmeier equation for the BK7 glass. Despite the fringe noise in the present measurement, there is a good agreement with the prediction. The slope, which is the group delay, can be extracted.

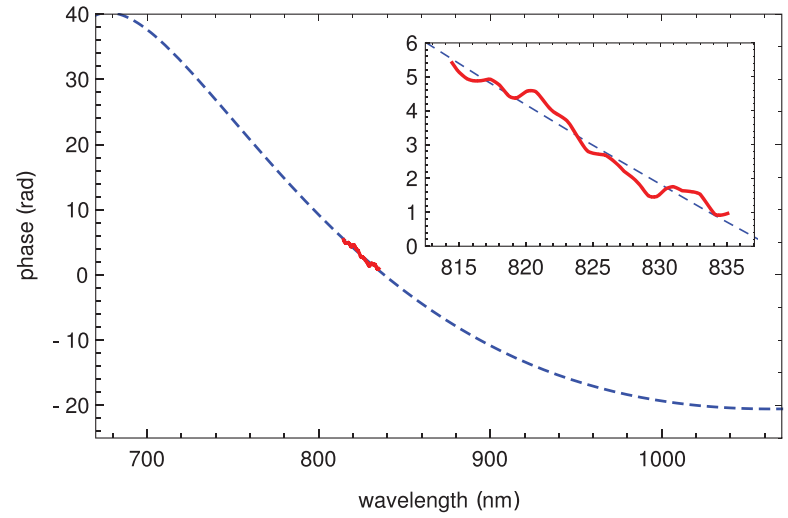

Figure 6. Phase change $\phi_{\mathrm{PDC}}$ for a slide of BK7 glass, obtained from its Sellmeier equation (dashed line) and measured from the observed interference fringes in the spectrum (solid line). The inset is a zoom into the measured data. The full band of shown wavelengths is potentially accessible with lower losses and higher-quality aperiodically poled crystals.

This method allows one potentially to perform a phase shift measurement in a very broad band of wavelengths with a precision at the Heisenberg limit. In our first experiment, because of many imperfections the band is limited to $20 \mathrm{~nm}$ and the precision is far from the limit. However, lowering losses and improving the quality of the aperiodic poling will provide an ultrabroadband phase measurement at a precision surpassing that of the interferometry with classical light. A similar recent experiment (16) reported a phase measurement in a band of $80 \mathrm{~nm}$ but in the low-gain regime, where at most one photon pair is produced for each pair of conjugated frequencies. 


\section{Conclusion}

We have considered, both theoretically and experimentally, a nonlinear interferometer formed by two aperiodically poled nonlinear crystals with a common quasi-monochromatic pump. Each crystal of this interferometer provides quasi-phase-matching for PDC in an ultrabroad range of frequencies, which can span the full octave of the visible light. On the theoretical side, we have analysed the operation of such an interferometer in terms of input and output squeezing angles, not used before. On the basis of the approximate 'quantum Rosenbluth formula' we have developed an analytic model for the field evolution in the interferometer. On the experimental side we have observed the interference fringes, caused by the dispersion of the generated PDC waves in both crystals forming the interferometer. We have also observed a displacement of the interference pattern caused by a sample between the crystals and inferred the phase shift within a band of $20 \mathrm{~nm}$. Our experimental observation is in a good agreement with the predictions of the developed model, up to imperfections of the samples. The obtained results pave the way to ultrabroadband nonlinear homodyning and to precise measurements of optical phase shifts in an octave-spanning spectral range.

\section{Acknowledgments}

We thank Andrea Cavanna for helpful discussions.

\section{Disclosure statement}

No potential conflict of interest was reported by the authors.

\section{Funding}

This work was supported by the Russian Science Foundation (RSF) (17-12-01134) and by the EPSRC (Engineering and Physical Sciences Research Council) via Grant No.
EP/N509759/1. IS acknowledges support from a Russell Trust Postgraduate Award.

\section{ORCID}

D. B. Horoshko (D) http://orcid.org/0000-0003-2550-948X

\section{References}

(1) Horoshko, D.B.; Kolobov, M.I. Phys. Rev. A. 2013, 88, 033806.

(2) Horoshko, D.B.; Kolobov, M.I. Phys. Rev. A. 2017, 95, 033837.

(3) Peřina, J. Sci. Rep. 2018, 8 (1), 15350.

(4) Chekhova, M.; Leuchs, G.; Zukowski, M. Opt. Comm. 2015, 337, 27-43.

(5) Caves, C.M. Phys. Rev. D 1981, 23, 1693-1708.

(6) Yurke, B.; McCall, S.L.; Klauder, J.R. Phys. Rev. A 1986, 33, 4033-4054.

(7) Chekhova, M.V.; Ou, Z.Y. Adv. Opt. Photon. 2016, 8 (1), 104-155.

(8) Chekhova, M.V.; Germanskiy, S.; Horoshko, D.B.; Kitaeva, G.K.; Kolobov, M.I.; Leuchs, G.; Phillips, C.R.; Prudkovskii, P.A. Opt. Lett. 2018, 43 (3), 375-378.

(9) Kolner, B.H. IEEE J. Quantum Elect. 1994, 30, 1951.

(10) Bennett, C.V.; Kolner, B.H. Opt. Lett. 1999, 24 (11), 783-785.

(11) Patera, G.; Shi, J.; Horoshko, D.B.; Kolobov, M.I. J. Opt. 2017, 19, 054001.

(12) Patera, G.; Horoshko, D.B.; Kolobov, M.I. Phys. Rev. A. 2018, 98, 053815.

(13) Medeiros de Araújo, R.; Roslund, J.; Cai, Y.; Ferrini, G.; Fabre, C.; Treps, N. Phys. Rev. A. 2014, 89, 053828.

(14) Shaked, Y.; Michael, Y.; Vered, R.Z.; Bello, L.; Rosenbluh, M.; Pe'er, A. Nature Commun. 2018, 9 (1), 609.

(15) Shaked, Y.; Pomerantz, R.; Vered, R.Z.; Pe'er, A. New J. Phys. 2014, 16, 053012.

(16) Riazi, A.; Zhu, E.Y.; Chen, C.; Gladyshev, A.V.; Kazansky, P.G.; Qian, L. Opt. Lett. 2019, 44, 1484.

(17) Kolobov, M.I. Rev. Mod. Phys. 1999, 71, 1539.

(18) Spasibko, K.Y.; Iskhakov, T.S.; Chekhova, M.V. Opt. Expr. 2012, 20 (7), 7507-7515. 